\title{
THE HILBERT TRANSFORM AND MAXIMAL FUNCTION FOR APPROXIMATELY HOMOGENEOUS CURVES
}

\author{
BY
}

DAVID A. WEINBERG

\begin{abstract}
Let $\mathcal{H}_{\gamma} f(x)=$ p.v. $\int_{-1}^{1} f(x-\gamma(t)) d t / t$ and $\mathscr{T}_{\gamma} f(x)=$ $\sup _{1>h>0} h^{-1} \int_{0}^{h}|f(x-\gamma(t))| d t$. It is proved that for $f \in \mathcal{S}\left(\mathbf{R}^{n}\right)$, the Schwartz class, and for an approximately homogeneous curve $\gamma(t) \in \mathbf{R}^{n},\left\|\mathcal{K}_{\gamma} f\right\|_{2}<C\|f\|_{2}$, $\left\|\Re_{\gamma} f\right\|_{2}<C\|f\|_{2}$.

A homogeneous curve is one which satisfies a differential equation $\gamma_{1}^{\prime}(t)=$ $(A / t) \gamma_{1}(t), 0<t<\infty$, where $A$ is a nonsingular matrix all of whose eigenvalues have positive real part. An approximately homogeneous curve $\gamma(t)$ has the form $\gamma_{1}(t)+\gamma_{2}(t)$, where $\gamma_{2}(t)$ is a carefully specified "error", such that $\gamma_{2}^{(t)}$ is also restricted for $j=2, \ldots, n+1$. The approximately homogeneous curves generalize the curves of standard type treated by Stein and Wainger.
\end{abstract}

I. Introduction. In this paper we shall be concerned with the following two operators.

$$
\mathcal{K}_{\gamma} f(x)=\text { p.v. } \int_{-\infty}^{\infty} f(x-\gamma(t)) \frac{d t}{t}
$$

and

$$
\mathfrak{R}_{\gamma} f(x)=\sup _{0<h<1} \frac{1}{h} \int_{0}^{h}|f(x-\gamma(t))| d t,
$$

where $\gamma$ is an appropriate curve in $\mathbf{R}^{n}$ and $f$ is in an appropriate space of functions. $\mathcal{H}_{\gamma}$ is an analogue of the familiar Hilbert transform. It originally arose in the study of parabolic partial differential equations. See [F]. If one suitably adapts Calderon's method of rotations to the study of certain of these equations, then the radial part of the integral essentially turns out to be the integral defining $\mathcal{K}_{\gamma}$. If it can be shown that $\mathfrak{N}_{\gamma}$ is a bounded operator (actually, only weak type is needed) then a version of the Fundamental Theorem of Calculus follows; namely

$$
\lim _{h \rightarrow 0} \frac{1}{h} \int_{0}^{h} f(x-\gamma(t)) d t=f(x) \text { a.e. }
$$

In the paper of Stein and Wainger [SW2], the following problem is posed on $\mathrm{p}$. 1290: Fix $p, 1<p<\infty$. Determine the class of curves $\gamma$ for which there are $L^{p}$ results for $\mathcal{H}$ and $\mathscr{N}$ corresponding to $\gamma$. I shall now sketch the history of work on this problem. The first result was the $L^{2}$ boundedness of $\mathcal{H}$ in the two-dimensional case $\gamma(t)=\left(t^{\alpha}, t^{\beta}\right)$. This was proved in 1966 by Fabes, who used complex

Received by the editors August 4, 1980.

1980 Mathematics Subject Classification. Primary 42-XX, 44XX; Secondary 42B20, 42B25.

Key words and phrases. Approximately homogeneous curve, nonisotropic dilation, Hilbert transform analogue, maximal function analogue, trigonometric estimate, $g$-function.

(C) 1981 American Mathematical Society 0002-9947/81/0000-0420/\$04.00 
integration. The difficulties of dealing with the saddle points in $n$-dimensions were by-passed in 1970 by Stein and Wainger, who used Van der Corput's Lemma to show that the multiplier $m$ is bounded, where $\left(\mathcal{H}_{\gamma} f\right)^{\wedge}=m \hat{f}$. They did this for the case $\gamma(t)=\left(t^{\alpha_{1}}, \ldots, t^{\alpha_{n}}\right)$. We now state

VAN DER CORPUT'S LemMA. Let $\left|d^{j} f(t) / d t^{j}\right| \geqslant \lambda>0$ for some positive integral value of $j$ and $b \leqslant t \leqslant d$. If $j=1$ assume in addition that $f^{\prime}(t)$ is monotonic. Then

$$
\left|\int_{b}^{d} \exp \{i f(t)\} d t\right| \leqslant \frac{c(j)}{\lambda^{1 / j}} .
$$

Nagel, Rivière, and Wainger proved in 1976 that $\mathcal{H}_{\gamma}: L^{p} \rightarrow L^{p}$ is bounded for $1<p<\infty$. In the same year, they proved the maximal theorem for the case $\gamma(t)=\left(t, t^{2}\right)$, i.e. that $\Re_{\gamma}: L^{p} \rightarrow L^{p}$ is bounded for $1<p<\infty$. See [NRW2]. Whereas the classical maximal functions are controlled by covering lemmas, these new maximal functions are controlled, at least partly, by the use of the Fourier transform. In accordance with this new idea, Stein introduced the $g$-function in 1976 to prove that $\Re_{\gamma}: L^{p} \rightarrow L^{p}$ is bounded for $1<p \leqslant \infty$ for homogeneous $\gamma$. See [S1]. Stein and Wainger proved in 1976 that $\mathfrak{N}_{\gamma}: L^{p} \rightarrow L^{p}$ is bounded for $1<p \leqslant \infty$, where $\gamma$ has nonzero curvature at 0 in the sense that $\left\{\gamma^{\prime}(0), \gamma^{\prime \prime}(0), \gamma^{\prime \prime \prime}(0), \ldots\right\}$ span the space that $\gamma$ lies in. Nagel and Wainger proved in 1976 that $\mathcal{H}_{\gamma}: L^{p} \rightarrow L^{p}$ is bounded for $\frac{5}{3}<p<\frac{5}{2}$, where $\gamma$ is a curve in two dimensions whose derivatives satisfy certain monotonicity conditions. See [NW]. This contrasts with the nonvanishing curvature condition because, for example, the curve $\left(t, t^{k} e^{-1 / t}\right)$ satisfies these monotonicity conditions, yet the derivatives at 0 span only the $x$-axis. In his thesis of 1980 , Bill Nestlerode obtained results for $\mathcal{H}_{\gamma}$ and $\mathfrak{T}_{\gamma}$, where $\gamma$ satisfies a certain generalization of these monotonicity conditions.

We now turn our attention to the curves considered in [SW2] since I will consider a generalization of these curves in the main body of this paper. Stein and Wainger consider two classes of curves-the homogeneous curves and the curves of standard type. The former class is homogeneous with respect to some nonisotropic dilations of the underlying $\mathbf{R}^{n}$. The nonisotropic dilations form a one-parameter family $\delta_{t}, t>0$, of linear transformations of $\mathbf{R}^{n}$ with the following properties:

(a) $\delta_{t} \delta_{s}=\delta_{t s}, t, s>0$,

(b) $\delta_{1}=$ identity

(c) $\delta_{t}(x) \rightarrow 0$ as $t \rightarrow 0$, for any $x \in \mathbf{R}^{n}$.

It follows from these properties that $\delta_{t}(x)=\exp (A \ln t)(x)$ for $t>0$, for some real matrix $A$ all of whose eigenvalues have positive real part.

A curve $\gamma(t), t>0$, is homogeneous with respect to $\delta_{t}$ if

$$
\gamma(t s)=\delta_{t}(s) \text { for } s, t>0,
$$

or equivalently, $\gamma(t)=\delta_{t} e, t>0$, where $e$ is a fixed vector in $\mathbf{R}^{n}$. Furthermore, $\gamma(t)$ is homogeneous with respect to $\delta_{t}$ if and only if $\gamma^{\prime}(t)=(A / t) \gamma(t)$. In order to develop a theory for $\mathcal{H}$, it is necessary to assume $\gamma(t)=\delta_{-t}(f)$, for $t<0$, where $f$ is some vector in $\mathbf{R}^{n}$, where the curves for positive $t$ and the curve for negative $t$ are compatible in the sense that the linear space spanned by $\{\gamma(t)\}_{t>0}$ equals the linear space spanned by $\{\gamma(t)\}_{t<0}$. 
Along with the dilations $\delta_{t}$, Stein and Wainger replace the Euclidean norm with a norm function $\rho(x)$, which is smooth and positive away from the origin and which is homogeneous with respect to the dilations $\delta_{t}$, i.e. $\rho\left(\delta_{t} x\right)=t \rho(x)$ for $t>0$. $\rho^{*}(\xi)$ will denote the norm function corresponding to the group $\delta_{t}^{*}=\exp \left(\ln t A^{*}\right)$. I shall describe some of the relevant properties of $\rho$ and $\delta_{t}$. For $x \neq 0$, there is a unique vector $w(x)$ such that $x=\delta_{\rho(x)} w(x)$, where $\langle w(x)\rangle=1$. Here, \langle\rangle is an appropriate norm defined on p. 1255 of [SW2]. From p. 1254 of [SW2] we have 1-6: For each $x$ with $|x|=1$

$$
c_{1} t^{\alpha_{1}}<\left|\delta_{t} x\right|<c_{2} t^{\alpha_{2}} \text { for } t \geqslant 1
$$

and

$$
c_{3} t^{\alpha_{3}}<\left|\delta_{t} x\right|<c_{4} t^{\alpha_{4}} \quad \text { for } t<1 ;
$$

here $c_{1}, c_{2}, c_{3}, c_{4}, \alpha_{1}, \alpha_{2}, \alpha_{3}, \alpha_{4}$ are some positive constants. From p. 1255 of [SW2] we have

Proposition 1-9. (i) $\rho\left(\delta_{t} x\right)=t \rho(x), t>0$;

(iv) $c_{1}|x|^{\alpha_{1}}<\rho(x)<c_{2}|x|^{\alpha_{2}}$ if $\rho(x) \geqslant 1$ and $c_{3}|x|^{\alpha_{3}}<\rho(x)<c_{4}|x|^{\alpha_{4}}$ if $\rho(x)<1$; where $c_{1}, c_{2}, c_{3}, c_{4}, \alpha_{1}, \alpha_{2}, \alpha_{3}, \alpha_{4}$ are some positive constants.

The other class of curves considered in [SW2] is the class of curves of standard type. A curve $\gamma(t)$ is of standard type if $\gamma(t)=\left(\gamma_{1}(t), \ldots, \gamma_{n}(t)\right)$, where $\gamma_{k}(t)=$ $t^{j_{k}} / j_{k}$ ! + higher terms, with $1 \leqslant j_{1}<j_{2}<\cdots<j_{n}$. If $\gamma(t)$ is of standard type, then we may associate to $\gamma$ the dilation group $\delta_{t}$ and norm function $\rho$ corresponding to the matrix $A=\operatorname{diag}\left(j_{1}, \ldots, j_{n}\right)$. Thus, $\gamma$ is approximately homogeneous with respect to diagonal matrices. In the main body of this paper, I wish to treat the more general class of approximately homogeneous curves $\gamma(t)=\gamma_{1}(t)+\gamma_{2}(t)$, where $\gamma_{1}(t)$ is a homogeneous curve corresponding to any real matrix $A$, all of whose eigenvalues have positive real part, and where $\gamma_{2}(t)$ is a carefully specified "error".

The methods used by Stein and Wainger to treat the curves of standard type do not apply to the more general case of approximately homogeneous curves. Thus, a new technique is required. In this paper, I will use this new technique to obtain a priori $L^{2}$ estimates for the Hilbert transform and maximal function.

This paper represents substantially the author's Ph.D. thesis at the University of Wisconsin. I wish to thank Professor Stephen Wainger, my thesis advisor, for his encouragement and many helpful suggestions.

II. A priori $L^{2}$ estimates for the Hilbert transform associated to approximately homogeneous curves. Let $\gamma_{1}(t)$ be a homogeneous curve in $\mathbf{R}^{n}$. Thus $\gamma_{1}^{\prime}(t)=$ $(A / t) \gamma_{1}(t)$, where all eigenvalues of $A$ have positive real part. We shall consider approximately homogeneous curves $\gamma(t)=\gamma_{1}(t)+\gamma_{2}(t)$. Furthermore, we will impose size restrictions on the derivatives of $\gamma_{2}(t)$ of orders $2, \ldots, n+1$. The errors $\gamma_{2}(t)$ are most naturally described with respect to a basis for $\mathbf{R}^{n}$ for which $A$ is in real canonical form.

We now describe the real canonical form of a matrix. See [CL]. Let $A$ be an $n \times n$ real matrix. Then there exists a real nonsingular matrix $P$ such that $\tilde{A}=P^{-1} A P$ has the real canonical form consisting of real square matrices 
$A_{1}, \ldots, A_{k}, B_{1}, \ldots, B_{m}$ down the main diagonal. Each $A_{j}$ has the form

$$
A_{j}=\left(\begin{array}{llllll}
S_{j} & 0_{2} & \cdots & 0_{2} & 0_{2} \\
E_{2} & & S_{j} & \cdots & 0_{2} & 0_{2} \\
0_{2} & & E_{2} & & 0_{2} & 0_{2} \\
& \cdots & & & \ldots & E_{2} \\
0_{2} & & 0_{2} & & S_{j}
\end{array}\right)
$$

where $0_{2}$ is the $2 \times 2$ zero matrix, $E_{2}$ is the $2 \times 2$ unit matrix, and

$$
S_{j}=\left(\begin{array}{cc}
\alpha_{j} & -\beta_{j} \\
\beta_{j} & \alpha_{j}
\end{array}\right)
$$

The $B_{j}$ have the form

$$
B_{j}=\left(\begin{array}{ccccc}
\lambda_{j} & 0 & \ldots & 0 & 0 \\
1 & \lambda_{j} & \ldots & 0 & 0 \\
0 & 1 & \ldots & 0 & 0 \\
0 & 0 & \ldots & 1 & \lambda_{j}
\end{array}\right) .
$$

$A_{j}$ corresponds to the eigenvalue pair $\alpha_{j} \pm i \beta_{j}$, while $B_{j}$ corresponds to the eigenvalue $\lambda_{j}$.

We will first prescribe the errors and prove the estimates for the case when $A$ is in real canonical form. We will then show that if $A$ is not in real canonical form, the estimates still hold, where the errors arise from a change of basis.

It will be convenient to refer to a parametrized version of our curves on the interval $(-\infty, \infty)$. A homogeneous curve on $(-\infty, \infty)$ satisfies $\psi_{1}^{\prime}=A \psi_{1}$ where $\psi_{1}(t)=\gamma_{1}\left(e^{t}\right)$. Modulo constants, the components of $\psi_{1}$ corresponding to a block $A_{j}$ are

$$
e^{\alpha_{j} t}\left(\cos \beta_{j} t, \sin \beta_{j} t, t \cos \beta_{j} t, t \sin \beta_{j} t, \ldots,\left(t^{l} / l !\right) \cos \beta_{j} t,\left(t^{l} / l !\right) \sin \beta_{j} t\right) .
$$

This is proved by induction on the size of the block. The errors in each of these components are assumed to be $o\left(e^{\left(\alpha_{j}+\varepsilon\right) t}\right), \varepsilon>0$. The errors in the derivatives of these components of order $2, \ldots, n+1$ are also assumed to be $o\left(e^{\left(a_{j}+e\right) t}\right)$. Modulo constants, the components of $\psi_{1}$ corresponding to a block $B_{j}$ are $e^{\lambda_{k}}\left(1, t, \ldots, t^{m} / m !\right), \lambda_{k}>0$. This is proved by induction on the size of the block. The errors $\psi_{2}(t)$ in these components of $\psi_{1}$ and in the components of $\psi_{1}^{(2)}, \ldots, \psi_{1}^{(n+1)}$ are assumed to be $o\left(e^{\left(\lambda_{*}+e\right) t}\right), \varepsilon>0$.

For an approximately homogeneous curve $\gamma(t)$, which is two-sided in the sense described in the introduction, set

$$
\mathcal{H}_{\varepsilon, 1} f(x)=\int_{\varepsilon<|t|<1} f(x-\gamma(t)) \frac{d t}{t},
$$

for $f \in \mathcal{S}$, where $\mathcal{S}$ is the Schwartz class. We shall prove

THEOREM 1. $\left\|\mathcal{H}_{\varepsilon, 1} f\right\|_{2} \leqslant c(\gamma)\|f\|_{2}$.

Also, as $\varepsilon \rightarrow 0, \mathcal{H}_{\varepsilon, 1}$ f converges in $L^{2}$ to an $L^{2}$ function $\mathcal{H} f$. Furthermore $\|\mathcal{H} f\|_{2}<$ $c(\gamma)\|f\|_{2}$. 
Proof. We first assume that $\gamma_{1}$ does not lie in a proper subspace.

$$
\widehat{\mathcal{H}_{e, 1}} f(\xi)=m_{e, 1}(\xi) \hat{f}(\xi)
$$

where $m_{e, 1}(\xi)=\int_{\varepsilon \leqslant|t| \leqslant 1} \exp (-2 \pi i \xi \cdot \gamma(t)) d t / t$. We must show that $m_{e, 1}(\xi)$ is uniformly bounded.

We shall require the following trigonometric estimate:

THEOREM 2. Let $\gamma(t)=\gamma_{1}(t)+\gamma_{2}(t)$, where $\gamma_{1}(t)$ is a homogeneous curve which does not lie in an affine hyperplane of $\mathbf{R}^{n} . \gamma_{2}(t)$ is the error such that $\gamma_{2}\left(e^{t}\right)=\psi_{2}(t)$, as described before. Then

$$
\left|\int_{b}^{d} \exp (i \xi \cdot \gamma(t h)) d t\right| \leqslant \frac{C}{\rho^{*}\left(\delta_{h}^{*} \xi\right)^{\sigma}},
$$

for some positive $C$ and $\sigma$, where $C$ remains bounded as long as $b$ and $d$ remain in $a$ compact subinterval of $(0, \infty)$ and $0 \leqslant h \leqslant h_{0}$, where $h_{0}$ may depend on $(b, d)$.

Proof. By changing variables and integrating by parts, we see that it suffices to prove

$$
\left|\int_{\alpha}^{\beta} \exp (i \xi \cdot \psi(t+h)) d t\right| \leqslant \frac{C}{\rho^{*}\left(\delta_{\left.e^{h} \xi\right)^{\circ}}\right.} .
$$

The idea of the proof is to use Van der Corput's Lemma and the fact that if $\xi \cdot \psi_{1}^{(j)}(t)$ is large where $\psi_{1}$ is homogeneous, then $\xi \cdot \psi^{(j)}(t)$ is still large enough, where $\psi$ includes the error terms. Thus, the trigonometric estimate is the part of our work which requires a restriction on the size of the errors in the derivatives, $\psi_{2}^{(J)}$, $j=2, \ldots, n+1$. We turn to the details.

We will use Van der Corput's Lemma to estimate the integral $\int_{\alpha}^{\beta} \exp (i f(t)) d t$, where $f(t)=\xi \cdot \psi(t+h)$. Stein and Wainger estimate an integral of this form for $f(t)=\xi \cdot \psi_{1}(t+h)$, where $\psi_{1}$ is a homogeneous curve. They use the derivatives of $f$ of orders $1, \ldots, n$. However, in order to apply Van der Corput's Lemma to $f^{\prime}$, it is necessary to check that $f^{\prime}$ is monotonic. This seems to cause difficulties when $\psi$ includes the error terms. In order to avoid these difficulties, I shall work with the derivatives of $f$ of orders $2, \ldots, n+1$. It turns out that since $\psi_{1}^{\prime}(t)=A \psi_{1}(t)$, the assumptions that $\gamma_{1}(t)$ not lie in any affine hyperplane and that $\gamma_{1}^{\prime}(t)$ not lie in any affine hyperplane are equivalent. We first redo part of an argument for homogeneous curves in [SW2], replacing the derivatives of orders $1, \ldots, n$ by the derivatives of orders $2, \ldots, n+1$. For the time being, $f(t)=\xi \cdot \psi_{1}(t+h)$.

We note that $f^{\prime \prime}(t)$ satisfies an $n$th order differential equation; for if $\sum_{j=0}^{n} c_{j} x^{j}$ is the characteristic polynomial of $A$,

$$
\sum_{j=0}^{n} c_{j} \frac{d^{j}}{d t^{j}}\left(f^{\prime \prime}(t)\right)=\sum_{j=0}^{n} c_{j}\left(\xi \cdot A^{j} \psi^{\prime \prime}(t)\right)=0 .
$$

Thus, if $f^{\prime \prime}\left(t_{0}\right)=\cdots=f^{(n+1)}\left(t_{0}\right)=0$, then

$$
\psi^{\prime \prime}\left(t_{0}\right) \cdot \xi=0, \ldots, \psi^{(n+1)}\left(t_{0}\right) \cdot \xi=0 .
$$

Thus, $A \psi^{\prime}\left(t_{0}\right) \cdot \xi=0, \ldots, A \psi^{(n)}\left(t_{0}\right) \cdot \xi=0$. Thus,

$$
\psi^{\prime}\left(t_{0}\right) \cdot A^{*} \xi=0, \ldots, \psi^{(n)}\left(t_{0}\right) \cdot A^{*} \xi=0 .
$$


$\therefore \psi^{\prime}(t) \cdot A^{*} \xi \equiv 0 . \therefore \psi(t) \cdot A^{*} \xi$ is constant, contradicting the assumption that $\gamma(t)$ not lie in any affine hyperplane. Now, by a compactness argument, we have, under the assumptions of Theorem 2 ,

$$
\sum_{j=2}^{n+1}\left|\frac{d^{j}}{d t^{j}}\left(\lambda \xi \cdot \psi_{1}(t)\right)\right| \geqslant c \lambda, \quad c>0,
$$

for $|\xi|=1, \lambda>0$, and $\alpha \leqslant t \leqslant \beta$. So Van der Corput's Lemma will prove

$$
\left|\int_{\alpha}^{\beta} \exp \left(i \lambda \xi \cdot \psi_{1}(t)\right) d t\right| \leqslant \frac{C}{\lambda^{1 / n+1}}
$$

if we can divide the interval $(\alpha, \beta)$ into a uniformly bounded (in $\lambda$ and $\xi$ ) number of subintervals in each of which a particular fixed one of the derivatives of orders $2, \ldots, n+1$ of $\lambda \xi \cdot \psi_{1}(t)$ is larger than or equal to the others. We first assume that none of the matrices $A, A^{2}, \ldots, A^{n}$ has 1 as an eigenvalue. Thus it suffices to prove:

(i) The number of zeros of $f^{(j)}(t)-f^{(k)}(t)$ is bounded for $\alpha<t \leqslant \beta, 2 \leqslant j<k$ $\leqslant n+1$, and $\xi$ on the unit sphere.

Proof. If the number of zeros of $f^{(j)}(t)-f^{(k)}(t)$ were not bounded, the argument principle would give a $\xi^{\prime} \neq 0$ such that $f^{(j)}(t)-f^{(k)}(t) \equiv 0$.

$\therefore \xi^{\prime} \cdot A^{j} \psi(t) \equiv \xi^{\prime} \cdot A^{k} \psi(t)$.

$\therefore\left(A^{* j} \xi^{\prime}-A^{* k} \xi^{\prime}\right) \cdot \psi(t) \equiv 0$.

$\therefore A^{* j} \xi^{\prime}-A^{* k} \xi^{\prime} \equiv 0$ from the hypothesis of the theorem.

$\therefore A^{*(k-j)} A^{* j} \xi^{\prime}=A^{* j} \xi^{\prime}$.

Since $A$ is nonsingular, $A^{* j} \xi^{\prime}$ is an eigenvector of $A^{*(k-j)}$ with eigenvalue 1 . However, we are assuming $A^{k-j}$ does not have 1 as an eigenvalue, giving us a contradiction. This proves (i).

The result is obtained without the hypothesis on the eigenvalues of $A, A^{2}, \ldots, A^{n}$ by changing variables.

Thus, we have proved that for $\psi_{1}$ homogeneous,

$$
\sum_{j=2}^{n+1}\left|\frac{d^{j}}{d t^{j}}\left(\xi \cdot \psi_{1}(t)\right)\right| \geqslant c|\xi|
$$

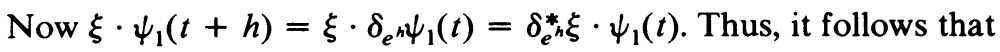

$$
\sum_{j=2}^{n+1}\left|\frac{d^{j}}{d t^{j}}\left(\xi \cdot \psi_{1}(t+h)\right)\right|=\sum_{j=2}^{n+1}\left|\frac{d^{j}}{d t^{j}}\left(\delta_{e^{n}}^{*} \xi \cdot \psi_{1}(t)\right)\right| \geqslant c\left|\delta_{e^{*}}^{*} \xi\right| \geqslant c_{1}\left[\rho^{*}\left(\delta_{e^{n}}^{*} \xi\right)\right]^{\tau_{1}}
$$

for $\alpha \leqslant t \leqslant \beta$ (since for the purposes of the trigonometric estimate we may assume $\left.\rho^{*}\left(\delta_{e^{\prime}}^{*} \xi\right) \geqslant 1\right)$.

We will prove that for $\psi(t)=\psi_{1}(t)+\psi_{2}(t)$, the ratio of $\left|\xi \cdot \psi_{2}^{(j)}(t)\right| /\left|\xi \cdot \psi_{1}^{(j)}(t)\right|$ is small enough so that $\left|\xi \cdot\left(\psi_{1}^{(j)}(t)+\psi_{2}^{(j)}(t)\right)\right|$ is still large if $\left|\xi \cdot \psi_{1}^{(j)}(t)\right|$ is large. More precisely, we will show that if

$$
\left|\xi \cdot \psi_{1}^{(j)}(t+h)\right| \geqslant\left(C_{1} / n\right)\left[\rho^{*}\left(\delta_{e^{*} \xi}^{*} \xi\right]^{\tau_{1}}\right.
$$

for $\alpha \leqslant t \leqslant \beta, h \leqslant h_{0}$, then

$$
\left|\psi^{(j)}(t)\right| \geqslant\left(C_{1} / 2 n\right)\left[\rho^{*}\left(\delta_{e}^{*} \xi\right)\right]^{\tau_{1}} .
$$


We wish to show that

$$
\frac{\sum_{j=2}^{n+1}\left|\xi \cdot \psi_{2}^{(j)}(t+h)\right|}{\sum_{j=2}^{n+1}\left|\xi \cdot \psi_{1}^{(j)}(t+h)\right|} \leqslant \frac{1}{2^{n+1}}
$$

for $\alpha \leqslant t \leqslant \beta, h \leqslant h_{0}$. Let

$$
\Psi_{1}(t+h)=\left(\begin{array}{ccc}
\psi_{11}^{\prime \prime}(t+h) & \psi_{12}^{\prime \prime}(t+h) & \cdots \\
\psi_{11}^{\prime \prime}(t+h) & \psi_{12}^{\prime \prime}(t+h) & \cdots \\
\cdots & & \cdots \\
\psi_{11}^{(n+1)}(t+h) & \psi_{12}^{(n+1)}(t+h) & \cdots
\end{array}\right)
$$

and

$$
\Psi_{2}(t+h)=\left(\begin{array}{ccc}
\psi_{21}^{\prime \prime}(t+h) & \psi_{22}^{\prime \prime}(t+h) & \cdots \\
\psi_{21}^{\prime \prime \prime}(t+h) & \psi_{22}^{\prime \prime \prime}(t+h) & \ldots \\
\cdots & & \cdots \\
\psi_{21}^{(n+1)}(t+h) & \psi_{22}^{(n+1)}(t+h) & \cdots
\end{array}\right)
$$

By the equivalence of norms on a finite dimensional space, it suffices to show that

$$
\frac{\left|\Psi_{2}(t+h) \xi\right|}{\left|\Psi_{1}(t+h) \xi\right|} \leqslant \frac{1}{2^{n+1}} \text {. }
$$

Let $\eta(t+h)=\Psi_{1}(t+h) \xi$. Then $\xi=\Psi_{1}^{-1}(t+h) \eta(t+h)$,

$$
\begin{gathered}
\frac{\left|\Psi_{2}(t+h) \xi\right|}{\left|\Psi_{1}(t+h) \xi\right|}=\frac{\left|\Psi_{2}(t+h) \Psi_{1}^{-1}(t+h) \eta(t+h)\right|}{|\eta(t+h)|} \\
\leqslant\left|\Psi_{2}(t+h) \Psi_{1}^{-1}(t+h)\right|, \\
\Psi_{1}(t+h)=\left(\begin{array}{cccc}
P_{11} e^{\alpha_{1}(t+h)} & P_{12} e^{\alpha_{2}(t+h)} & \cdots & P_{1 n} e^{\alpha_{n}(t+h)} \\
P_{21} e^{\alpha_{1}(t+h)} & P_{22} e^{\alpha_{2}(t+h)} & \cdots & P_{2 n} e^{\alpha_{n}(t+h)} \\
P_{n 1} e^{\alpha_{1}(t+h)} & P_{n 2} e^{\alpha_{2}(t+h)} & \cdots & P_{n n} e^{\alpha_{n}(t+h)}
\end{array}\right),
\end{gathered}
$$

where $P_{i j}(t)=O\left(t^{n}\right)$. By Theorem 7.3, p. 28 in [CL], det $\Psi_{1}(t+h)=C e^{(\operatorname{tr} A)(t+h)}$, $\Psi_{1}^{-1}(t+h)$

$$
=\frac{1}{C e^{(\operatorname{tr} A)(t+h)}}\left(\begin{array}{ccc}
Q_{11} e^{\left(\alpha_{2}+\cdots+\alpha_{n}\right)(t+h)} & Q_{12} e^{\left(\alpha_{2}+\cdots+\alpha_{n}\right)(t+h)} & \cdots \\
\cdots & Q_{n 2} e^{\left(\alpha_{1}+\cdots+\alpha_{n-1}\right)(t+h)} & \cdots
\end{array}\right),
$$

where $Q_{i j}=O\left(t^{n^{2}-n}\right)$.

$$
\Psi_{2}(t+h)=\left(\begin{array}{lll}
R_{11} e^{\left(\alpha_{1}+e\right)(t+h)} & \cdots & R_{1 n} e^{\left(\alpha_{n}+e\right)(t+h)} \\
R_{n 1} e^{\left(\alpha_{1}+\varepsilon\right)(t+h)} & \cdots & R_{n n} e^{\left(\alpha_{n}+e\right)(t+h)}
\end{array}\right),
$$

where $R_{i j}=O(1)$. 


$$
\Psi_{2} \Psi_{1}^{-1}(t+h)=\left(\begin{array}{lll}
S_{11} e^{\varepsilon(t+h)} & \cdots & S_{1 n} e^{\varepsilon(t+h)} \\
S_{n 1} e^{\varepsilon(t+h)} & \cdots & S_{n n} e^{\varepsilon(t+h)}
\end{array}\right),
$$

where $S_{i j}=O\left(t^{n^{2}-n}\right)$.

$$
\therefore\left|\Psi_{2} \Psi_{1}^{-1}\right| \leqslant 1 / 2^{n+1} \text { for } \alpha \leqslant t \leqslant \beta, h \leqslant h_{0},
$$

where $h_{0}$ is sufficiently small. This proves that

$$
\left|\int_{\alpha}^{\beta} \exp (i \xi \cdot \psi(t+h)) d t\right| \leqslant \frac{C}{\rho^{*}\left(\delta_{\left.e^{*} \xi\right)^{\sigma}}^{\sigma}\right.},
$$

and completes the proof of Theorem 2 .

We now divide the integral for $m_{\varepsilon, 1}(\xi)$ into $t<1 / \rho^{*}(\xi)$ and $t \geqslant 1 / \rho^{*}(\xi)$. Thus, for $t_{0}$ sufficiently small,

$$
\begin{aligned}
\left|\int_{\substack{|t|<1 / \rho^{*}(\xi) \\
|t|<t_{0}}} \exp (i \xi \cdot \gamma(t)) \frac{d t}{t}\right| & \leqslant \int_{\substack{|t|<1 / \rho^{*}(\xi) \\
|t|<t_{0}}}|\exp (i \xi \cdot \gamma(t))-1| \frac{d t}{t} \\
& \leqslant \int_{\substack{|t|<1 / \rho^{*}(\xi) \\
|t|<t_{0}}}|\xi \cdot \gamma(t)| \frac{d t}{t} \\
& \leqslant \int_{\substack{|t|<1 / \rho^{*}(\xi) \\
|t|<t_{0}}}\left(\left|\xi \cdot \gamma_{1}(t)\right|+\left|\xi \cdot \gamma_{2}(t)\right|\right) \frac{d t}{|t|} .
\end{aligned}
$$

Now, by changing variables and using homogeneity,

$$
\int_{|t|<1 / \rho^{*}(\xi)}\left|\xi \cdot \gamma_{1}(t)\right| \frac{d t}{|t|} \leqslant C .
$$

So consider $\int_{|t| \leqslant 1 / \rho^{*}(\xi) ;|t| \leqslant t_{0}}\left|\xi \cdot \gamma_{2}(t)\right| d t /|t|$. We are now entering the part of the proof which requires the size restriction on the "error term" in the curve itself, $\gamma_{2}(t)$.

$$
\begin{aligned}
\int_{|t|<1 / \rho^{*}(\xi)}\left|\xi \cdot \gamma_{2}(t)\right| \frac{d t}{|t|} & =\int_{|t|<1 / \rho^{*}(\xi)}\left|\delta_{\rho^{*}(\xi)}^{*} w^{*}(\xi) \cdot \gamma_{2}(t)\right| \frac{d t}{|t|} \\
& =\int_{|t|<1 / \rho^{*}(\xi)}\left|w^{*}(\xi) \cdot \delta_{\rho^{*}(\xi)} \gamma_{2}(t)\right| \frac{d t}{|t|} \quad S=t \rho^{*}(\xi), \\
& =\int_{|S|<1}\left|w^{*}(\xi) \cdot \delta_{\rho^{*}(\xi)} \gamma_{2}\left(\frac{S}{\rho^{*}(\xi)}\right)\right| \frac{d S}{|S|} \\
& <C \int_{|S|<1 \mid}\left|\delta_{\rho^{*}(\xi)} \gamma_{2}\left(\frac{S}{\rho^{*}(\xi)}\right)\right| \frac{d S}{|S|}
\end{aligned}
$$

Consider $\delta_{\lambda} \gamma_{2}(t / \lambda)$ for $\lambda>1 . \delta_{\lambda}=e^{A \ln \lambda}$. If $A$ is in real canonical form with blocks $A_{j}$ and $B_{j}$, then $e^{A t}$ has blocks of the following form. 
Corresponding to the $A_{j}$ we have

$$
e^{\alpha t}\left(\begin{array}{ccccc}
\cos \beta t & -\sin \beta t & & 0 \\
\sin \beta t & \cos \beta t & & & \\
t \cos \beta t & -t \sin \beta t & \cos \beta t & -\sin \beta t & \\
t \sin \beta t & t \cos \beta t & \sin \beta t & \cos \beta t & \\
\frac{t^{2}}{2 !} \cos \beta t & -\frac{t^{2}}{2 !} \sin \beta t & & & \\
\frac{t^{2}}{2 !} \sin \beta t & \frac{t^{2}}{2 !} \cos \beta t & \ddots & \ddots & \\
\vdots & & &
\end{array}\right)
$$

and corresponding to the $B_{j}$ we have

$$
e^{\alpha t}\left(\begin{array}{ccccc}
1 & 0 & 0 & \ldots & \ldots \\
t & 1 & 0 & \ldots & \ldots \\
\frac{t^{2}}{2 !} & t & 1 & 0 & \ldots \\
\frac{t^{3}}{3 !} & \frac{t^{2}}{2 !} & t & 1 & \ldots \\
\vdots & & & &
\end{array}\right)
$$

Thus, a typical coordinate of $\delta_{\lambda} \gamma_{2}(t / \lambda)$ looks like

$$
\lambda^{\alpha}\left(\frac{t}{\lambda}\right)^{\alpha+\varepsilon} d_{\alpha}=\frac{t^{\alpha+\varepsilon}}{\lambda^{e}} d_{\alpha}
$$

where $d_{\alpha}=O\left((\ln \lambda)^{n}\right) . \therefore$ since

$$
\int_{|S|<1} \frac{\left|S^{\alpha+\varepsilon} O\left((\ln \lambda)^{n}\right)\right|}{\lambda^{\varepsilon}|S|} d S<\infty \text { for } \alpha>0, \lambda>1,
$$

we have that for $\rho^{*}(\xi)>1,|t| \leqslant t_{0}$,

$$
\int_{|S|<1}\left|\delta_{\rho^{*}(\xi)} \gamma_{2}\left(\frac{S}{\rho^{*}(\xi)}\right)\right| \frac{d S}{|S|} \leqslant C .
$$

Now let $\eta$ be a small number depending on $\gamma$, and let $t_{0}$ be a small number depending on $\gamma$ and $\eta$. Then if $1<\alpha \leqslant \beta \leqslant 2 \alpha$,

$$
\begin{aligned}
\left|\int_{\alpha / \rho^{*}(\xi)<|t|<\beta / \rho^{*}(\xi)} \exp (i \xi \cdot \gamma(t)) \frac{d t}{t}\right|=\left|\int_{\substack{1<|t|<\beta / \alpha \\
|t|<t_{0} \rho^{*}(\xi) / \alpha}} \exp \left(i \xi \cdot \gamma\left(\frac{t \alpha}{\rho^{*}(\xi)}\right)\right) \frac{d t}{t}\right| \\
=\left|\int_{\eta<|t|<\eta \beta / \alpha} \exp \left(i \xi \cdot \gamma\left(\frac{1}{\eta} t \frac{\alpha}{\rho^{*}(\xi)}\right)\right) \frac{d t}{t}\right| \leqslant C_{\eta} \frac{1}{\alpha^{\sigma}}
\end{aligned}
$$


if $\eta$ is sufficiently small and $t_{0}$ is sufficiently small (since $\alpha / \rho^{*}(\xi)<t_{0}$ ). Adding the terms shows $\left|m_{\varepsilon, t_{0}}(\xi)\right| \leqslant c(\gamma)$. Having fixed $t_{0}$,

$$
\left|\int_{t_{0}<|t|<1} \exp (i \xi \cdot \gamma(t)) \frac{d t}{t}\right| \leqslant C .
$$

The proof when $\gamma_{1}$ lies in a proper subspace is completed as in [SW2] at the bottom of p. 1262.

We now show that it suffices to consider $A$ in real canonical form. Let $\tilde{A}=P^{-1} A P$ be in real canonical form.

$$
\begin{aligned}
& \gamma_{1}(t)=e^{A \ln t} \vec{v}_{0}, \\
& \tilde{\gamma}_{1}(t)=e^{\tilde{A} \ln t} \vec{w}_{0}=e^{P^{-1} A P \ln t} \vec{w}_{0}=P^{-1} e^{A \ln t} P \vec{w}_{0} .
\end{aligned}
$$

Choose $\vec{w}_{0}$ such that $P \vec{w}_{0}=\vec{v}_{0}$. Then $P \tilde{\gamma}_{1}(t)=e^{A \ln t} \vec{v}_{0}=\gamma_{1}(t)$. We also let $\gamma_{2}(t)=P \tilde{\gamma}_{2}(t)$, where $\tilde{\gamma}_{2}(t)$ is a suitable error for $\tilde{\gamma}_{1}(t)$. Then

$$
\begin{aligned}
\int \exp (i \xi \cdot \gamma(t)) \frac{d t}{t} & =\int \exp (i \xi \cdot P \tilde{\gamma}(t)) \frac{d t}{t} \\
& =\int \exp \left(i P^{*} \xi \cdot \tilde{\gamma}(t)\right) \frac{d t}{t}
\end{aligned}
$$

and

$$
\left|\int \exp \left(i P^{*} \xi \cdot \tilde{\gamma}(t)\right) \frac{d t}{t}\right| \leqslant \frac{C}{\rho^{*}\left(P^{*} \xi\right)^{\sigma}} .
$$

But $\rho^{*}\left(P^{*} \xi\right) \geqslant C_{1}\left|P^{*} \xi\right|^{\alpha_{1}} \geqslant C_{1}\left(C_{2}|\xi|\right)^{\alpha_{1}} \geqslant C_{1} C_{2}^{\alpha_{1}} C_{3} \rho^{*}(\xi)^{\alpha_{1} \alpha_{2}}$ provided that $\rho^{*}\left(P^{*} \xi\right) \geqslant$ 1 and $\rho^{*}(\xi) \geqslant 1$.

$$
\therefore\left|\int \exp (i \xi \cdot \gamma(t)) \frac{d t}{t}\right| \leqslant \frac{C}{\rho^{*}(\xi)^{\sigma}} .
$$

This completes the proof of Theorem 1 .

III. A priori $L^{2}$ estimates for the maximal function associated to approximately homogeneous curves. Let $\gamma(t)$ be an approximately homogeneous curve defined for $t \geqslant 0$. For $f$ in $\mathcal{S}$ we set

$$
\mathscr{T} f(x)=\sup _{1>h>0} \frac{1}{h}\left|\int_{0}^{h} f(x-\gamma(t)) d t\right| .
$$

THEOREM 3. $\|\mathfrak{N} f\|_{2} \leqslant c(\gamma)\|f\|_{2}$.

As in [SW2] this theorem will be proved by obtaining estimates for appropriate $g$ functions. We set

$$
N_{h} f(x)=\frac{1}{h} \int_{h}^{2 h} f(x-\gamma(t)) d t .
$$

Let $\varphi$ be a $C_{0}^{\infty}$ function with $\hat{\varphi}(0)=1$, and $\varphi_{h}(x)=\left(\operatorname{det} \delta_{h}\right)^{-1} \varphi\left(\delta_{h}^{-1} x\right)$, for the appropriate dilation group. If $\gamma(t)$ is approximately homogeneous and $f$ is in $\mathcal{S}$, we define

$$
g^{2} f(x)=\int_{0}^{1} \frac{d h}{h}\left|N_{h} f(x)-\varphi_{h} * f(x)\right|^{2}
$$


Here the underlying group of dilations is that associated with the homogeneous curve $\gamma_{1}(t)$, where $\gamma(t)=\gamma_{1}(t)+\gamma_{2}(t)$.

THEOREM 4. If $\gamma(t)$ is approximately homogeneous, then $\|g(f)\|_{2} \leqslant c(\gamma)\|f\|_{2}$.

The proof that Theorem 4 implies Theorem 3 is exactly the same as the argument in [SW2] on p. 1265.

Proof OF THEOREM 4.

$$
\begin{aligned}
\|g(f)\|_{2}^{2} & =\int_{0}^{1} \frac{d h}{h}\left\|N_{h} f(x)-\varphi_{h} * f(x)\right\|_{2}^{2} \\
& =\int_{0}^{1} \frac{d h}{h}\left\|N_{h} f(\xi)-\varphi_{h} * f(\xi)\right\|_{2}^{2} .
\end{aligned}
$$

Now $\left(\varphi_{h} * f\right)^{\wedge}(\xi)=\hat{\varphi}\left(\delta_{h}^{*} \xi\right) \hat{f}(\xi)$, and a simple calculation shows $\left(N_{h} f\right)^{\wedge}(\xi)=$ $m_{h}(\xi) \hat{f}(\xi)$, where

$$
m_{h}(\xi)=\frac{1}{h} \int_{h}^{2 h} \exp (-i \xi \cdot \gamma(t)) d t=\int_{1}^{2} \exp (-i \xi \cdot \gamma(t h)) d t
$$

Thus,

$$
\|g(f)\|_{2}^{2}=\int|\hat{f}(\xi)|^{2} \int_{0}^{1}\left|m_{h}(\xi)-\hat{\varphi}\left(\delta_{h}^{*} \xi\right)\right|^{2} \frac{d h}{h} d \xi .
$$

Hence, it suffices to show that

$$
\int_{0}^{1}\left|m_{h}(\xi)-\hat{\varphi}\left(\delta_{h}^{*} \xi\right)\right|^{2} \frac{d h}{h} \leqslant C .
$$

In the integral (M-1) only the part where $h$ is small is critical. If we divide that part into two further parts (where $h<1 / \rho^{*}(\xi)$, and $h \geqslant 1 / \rho^{*}(\xi)$ ), we see that (M-1) follows from the four estimates

$$
\begin{aligned}
\left|1-m_{h}(\xi)\right| & \leqslant C\left|\delta_{h}^{*} \xi\right|^{\tau}, \\
\left|1-\hat{\varphi}\left(\delta_{h}^{*} \xi\right)\right| & \leqslant C\left|\delta_{h}^{*} \xi\right|^{\tau}, \\
\left|\hat{\varphi}\left(\sigma_{h}^{*} \xi\right)\right| & \leqslant C\left|\delta_{h}^{*} \xi\right|^{-\sigma}, \\
\left|m_{h}(\xi)\right| & \leqslant C\left|\delta_{h}^{*} \xi\right|^{-\sigma},
\end{aligned}
$$

for small $h$ and some $\sigma, \tau>0$. (M-3) and (M-4) are easy. (M-5) follows from the trigonometric estimate proved in §II. Here is the argument for (M-2): By an argument similar to the one at the end of $\S$ II, we may assume that $A$ is in real canonical form, where $\gamma_{1}^{\prime}(t)=A \gamma_{1}(t) / t$.

$$
\begin{aligned}
\left|1-m_{h}(\xi)\right| & =\left|1-\int_{1}^{2} \exp (-i \xi \cdot \gamma(t h)) d t\right|=\left|\int_{1}^{2} 1-\exp (-i \xi \cdot \gamma(t h)) d t\right| \\
& \leqslant \int_{1}^{2}|1-\exp (-i \xi \cdot \gamma(t h))| d t \leqslant \int_{1}^{2}|\xi \cdot \gamma(t h)| d t \\
& \leqslant \int_{1}^{2}\left|\xi \cdot \gamma_{1}(t h)\right|+\left|\xi \cdot \gamma_{2}(t h)\right| d t
\end{aligned}
$$


Now

$$
\begin{aligned}
& \int_{1}^{2}\left|\xi \cdot \gamma_{1}(t h)\right| d t=\int_{1}^{2}\left|\xi \cdot \delta_{h} \gamma_{1}(t)\right| d t \\
& =\int_{1}^{2}\left|\delta_{h}^{*} \xi \cdot \gamma_{1}(t)\right| d t \leqslant C\left|\delta_{h}^{*} \xi\right|, \\
& \int_{1}^{2}\left|\xi \cdot \gamma_{2}(t h)\right| d t=\int_{h}^{2 h}\left|\delta_{\rho^{*}(\xi)}^{*} w^{*}(\xi) \cdot \gamma_{2}(t)\right| d t \\
& =\int_{h}^{2 h}\left|w^{*}(\xi) \cdot \delta_{\rho^{*}(\xi)} \gamma_{2}(t)\right| d t \\
& =\int_{h \rho^{*}(\xi)}^{2 h \rho^{*}(\xi)}\left|w^{*}(\xi) \cdot \delta_{\rho^{*}(\xi)} \gamma_{2}\left(\frac{S}{\rho^{*}(\xi)}\right)\right| \frac{d S}{\rho^{*}(\xi)} \\
& \text { (obtained by changing variables: } t=S / \rho^{*}(\xi) \text { ) } \\
& \leqslant C \int_{h \rho^{*}(\xi)}^{2 h \rho^{*}(\xi)}\left|\delta_{\rho^{*}(\xi)} \gamma_{2}\left(\frac{S}{\rho^{*}(\xi)}\right)\right| \frac{d S}{\rho^{*}(\xi)} \text {. }
\end{aligned}
$$

A typical coordinate of $\delta_{\rho^{*}(\xi)} \gamma_{2}\left(S / \rho^{*}(\xi)\right)$ looks like $\left(\rho^{*}(\xi)\right)^{\alpha}\left(S / \rho^{*}(\xi)\right)^{\alpha+\varepsilon} d_{\alpha}$, where $d_{\alpha}=O\left(\left(\ln \rho^{*}(\xi)\right)^{n}\right) . \quad \therefore$ the latter integral

$$
\begin{aligned}
& \leqslant C \int_{h \rho^{*}(\xi)}^{2 h \rho^{*}(\xi)} \frac{S^{\alpha+\varepsilon} d_{\alpha}}{\left(\rho^{*}(\xi)\right)^{\varepsilon+1}} d S \\
& \leqslant\left. C \frac{S^{\alpha+\varepsilon+1}}{\left(\rho^{*}(\xi)\right)^{\varepsilon+1}}\right|_{h \rho^{*}(\xi)} ^{2 h \rho^{*}(\xi)} \leqslant c h^{1+\varepsilon}\left(h \rho^{*}(\xi)\right)^{\alpha},
\end{aligned}
$$

and since $h \leqslant 1,(\mathrm{M}-2)$ is proved. This completes the proof of Theorem 4 , and hence of Theorem 3 .

\section{REFERENCES}

[CL] E. Coddington and N. Levinson, Theory of ordinary differential equations, McGraw-Hill, New York, 1955.

[F] E. Fabes, Singular integrals and partial differential equations of parabolic type, Studia Math. 28 (1966), 81-131.

[NRW1] A. Nagel, N. M. Rivière and S. Wainger, On Hilbert transforms along curves, Bull. Amer. Math. Soc. 8 (1974), 106-108.

[NRW2] __, On Hilbert transforms along curves. II, Amer. J. Math. 98 (1976), 395-403.

[NW] A. Nagel and S. Wainger, Hilbert transforms associated with plane curves, Trans. Amer. Math. Soc. 223 (1976), 235-252.

[N] W. Nestlerode, $L^{2}$ estimates for singular integrals and maximal functions associated with highly monotone curves, Ph.D. Dissertation, University of Wisconsin, Madison, Wisc., 1980.

[R] N. M. Rivière, Singular integrals and multiplier operators, Ark. Mat. 9 (1971), 243-278.

[S1] E. M. Stein, Maximal functions: homogeneous curves, Proc. Nat. Acad. Sci. U.S.A. 73 (1976), 2176-2177.

[S2] __ Singular integrals and differentiability properties of functions, Princeton Univ. Press, Princeton, N.J., 1970.

[SW1] E. M. Stein and S. Wainger, The estimation of an integral arising in multiplier transformations, Studia Math. 35 (1970), 101-104. , Problems in harmonic analysis related to curvature, Bull. Amer. Math. Soc. 84 (1978),

[SWe] E. M. Stein and G. Weiss, Introduction to Fourier analysis on Euclidean spaces, Princeton Univ. Press, Princeton, N.J., 1971.

Department of Mathematics, Texas Tech University, Lubbock, TeXas 79406 